\title{
Cognitive Function Assessment in Patients on Moderate- or High-Intensity Statin Therapy
}

\author{
Satyajeet Roya, d, Daniel Hyman a, Srinivas Ayyala ${ }^{a}$, Aditya Bakhshia , Sang Hoon Kimª, \\ Nancy Anoruo ${ }^{\mathrm{b}}$, Joshua Weinstock ${ }^{\mathrm{a}}$, Ayobamidele Balogun ${ }^{\mathrm{a}}$, Michelle D'Souza ${ }^{\mathrm{a}}$, \\ Nika Filatova ${ }^{\mathrm{a}}$, Jesus Penabad ${ }^{\mathrm{a}}$, Pratik Shah ${ }^{\mathrm{a}}$, Christopher Perez ${ }^{\mathrm{a}}$, \\ Anita Mehta ${ }^{a}$, Krystal Hunter ${ }^{\mathrm{c}}$
}

\begin{abstract}
Background: Cardiovascular diseases are the leading cause of death in the USA. Statin therapy reduces cardiovascular events significantly. Cognitive impairment has been reported with statin therapy but there is a lack of consensus. We analyzed the cognitive functions of adult patients who were on moderate-intensity statin therapy (MIST) or high-intensity statin therapy (HIST).
\end{abstract}

Methods: A total of 213 patients underwent cognitive assessment testing. Cognitive function scores were correlated with the durations of statin therapy, age, and level of education by using Pearson correlation. Independent $t$-test was used to compare the mean cognitive function score to the gender, race, type of statin therapy, and comorbid conditions.

Results: Mean age of all the patients was 55.4 years. Majority of the patients $(66.2 \%)$ were on MIST while the rest $(33.8 \%)$ were on HIST. Cognitive impairment was observed in $17.8 \%$ of the studied patients. A total of $41.7 \%$ of the patients in the HIST group and $5.7 \%$ in the MIST group had cognitive impairment $(\mathrm{P}<0.001)$. There was no correlation between cognitive function score and age $(r=-0.106)$, weakly positive correlation between the level of education and cognitive function score $(r=0.252)$, and weakly negative correlation between the duration of statin therapy and cognitive function score $(\mathrm{r}=$ -0.283 ). In the group of patients on HIST with cognitive impairment, the proportion of patients on atorvastatin $40-80 \mathrm{mg}$ was significantly higher than the proportion of patients on rosuvastatin $20-40 \mathrm{mg}$ $(66.7 \%$ vs. $33.3 \%$; $\mathrm{P}<0.05)$. In the group of patients on MIST with

Manuscript submitted March 21, 2020, accepted March 28, 2020

aDepartment of Medicine, Cooper Medical School of Rowan University, Cooper University Health Care, Camden, NJ, USA

${ }^{b}$ Department of Medicine, University of Massachusetts Medical SchoolUMASS Memorial Medical Center, Worcester, MA, USA

${ }^{\mathrm{c}}$ Cooper Research Institute, Cooper Medical School of Rowan University, Camden, NJ, USA

${ }^{\mathrm{d} C}$ Corresponding Author: Satyajeet Roy, Department of Medicine, Cooper Medical School of Rowan University, Cooper University Health Care, 1103 North Kings Highway, Suite 203, Cherry Hill, NJ 08034, USA.

Email: roy-satyajeet@cooperhealth.edu

doi: https://doi.org/10.14740/jocmr4144 cognitive impairment, atorvastatin 10 - $20 \mathrm{mg}$ was the most commonly used statin therapy (50\%), followed by rosuvastatin $10 \mathrm{mg}(25 \%)$, simvastatin 20 - $40 \mathrm{mg}(12.5 \%)$ and pravastatin 40 - $80 \mathrm{mg}(12.5 \%)$.

Conclusions: We found a significantly higher association of cognitive impairment in patients who were on MIST or HIST compared to the general population. We found no correlation between cognitive function score and age, weakly positive correlation between the level of education and cognitive function score, and weakly negative correlation between the duration of statin therapy and cognitive function score. HIST was associated with a higher frequency of cognitive impairment compared to the MIST.

Keywords: Cognitive function; Cognitive impairment; Dementia; Statin therapy

\section{Introduction}

Atherosclerotic cardiovascular disease is the leading cause of death, both nationally and worldwide [1]. Overwhelming evidence supports that treatment with a statin (3-hydroxy-3-methyl-glutaryl-CoA reductase inhibitors) reduces cardiovascular events [2]. In the last decade, the number of adults on statin therapy has gradually increased [3]. This is in part due to inclusion of a substantial number of people who are qualified to be included in the statin benefit groups by following the 2013 American College of Cardiology/American Heart Association guidelines [4]. According to a cohort study, the use of statins has increased from $17.9 \%$ in $2002-2003$ to $27.8 \%$ in 2012 2013 among adults aged 40 years and older [5]. It is, therefore, important to be aware of all of the potential benefits and risks associated with the use of statins.

In 2012, the US Food and Drug Administration (FDA) released a new warning regarding the use of statin therapy and cognitive impairment by stating "certain cognitive (brainrelated) effects have been reported with statin use" [6]. The basis of the report was a review of the multiple studies and clinical trials on statins that included assessment of cognitive function [7-18]. In 2016, the FDA announced: "Memory loss and confusion have been reported with statin use. These reported events were generally not serious and went away once 
the drug was no longer being taken" [19]. Human brain is the most cholesterol-rich organ which contains about $20 \%$ of the total cholesterol in the human body [20]. Cholesterol is an important constituent of the myelin sheaths and the plasma membranes of astrocytes and neurons. Cholesterol is imperative for signal transmission in synapses within the central nervous system [20]. Low levels of cholesterol may have a negative influence on the composition and functioning of the neurons.

Many studies have reported association between the use of statins to lower circulating cholesterol levels and decline in neurocognitive function [7-9, 20-23]. On the contrary, there are many studies that have shown either no association between the use of statins and cognitive changes [24-27], or a beneficial neuroprotective role attributable to the statin use [28-36].

It is clear that there has been a lack of consensus among the available studies evaluating the impact of statin use on the cognitive function. The aims and objectives of our study were to analyze the cognitive functions of adult patients who were on a moderate-intensity statin therapy (MIST) or a high-intensity statin therapy (HIST), and to compare the proportion of patients with cognitive impairment in this group with that of the general population in the USA in order to assess if there was a higher prevalence of cognitive impairment among persons on statin therapy.

\section{Materials and Methods}

\section{Study selection}

This was a prospective study which included single-arm assessment of cognitive function in patients who were on MIST or HIST. Patient enrollment started in January 2017 and completed in February 2020. The study was reviewed and approved by the Institutional Review Board of the Cooper University Health Care (CUHC), Camden, NJ, USA. This study was fully compliant with the ethical standards set forth by the CUHC institutional review board.

Adult patients aged 18 years and older who presented in our internal medicine offices and who were either on MIST or HIST were approached by the study investigators for enrollment during their scheduled routine office visits. All patients received a description of the study and they were informed about the purpose, risks, benefits, alternatives, and follow-ups. Informed consents were obtained from each patient who decided to participate in the study. A one-time in-office cognitive function was assessed on each patient as per the established assessment tool Modified Mini-Mental State Examination (3MS) [37], which is a questionnaire based cognitive assessment tool. The study investigators also reviewed the electronic medical records of the patients for data collection.

The inclusion criteria were English language speaking patients aged 18 years or older, who were taking a moderateintensity or high-intensity statin, and who were willing to give consent to participate in the study. The exclusion criteria were patients with established diagnosis of dementia due to any cause, patients who were not self-administering medications, patients who could not communicate in English language, pa- tients who had advanced comorbid medical condition(s) that could have affected their cognitive function, such as advanced neurological condition (e.g. cerebrovascular accident, Parkinson's disease, multiple sclerosis), advanced cardiac condition with poor performance state (e.g. New York Heart Association (NYHA) III or IV congestive heart failure, cardiomyopathy with left ventricular ejection fraction of less than $40 \%$ ), advanced pulmonary disorder (e.g. chronic obstructive or restrictive airway disease requiring ambulatory oxygen therapy), endstage renal disease on hemodialysis, end-stage liver disease (e.g. cirrhosis), hematological disorders leading to severe anemia (hemoglobin less than $9.0 \mathrm{~g} / \mathrm{dL}$ ), advanced uncontrolled rheumatological disorder (e.g. rheumatoid arthritis, systemic lupus erythematosus, osteoarthritis), advanced multisystem disorder (e.g. sarcoidosis), and patients with factors limiting their cognitive assessment (e.g. developmental disorders, mental retardation, and uncontrolled psychiatric disorders).

\section{Data collection}

After obtaining informed consents the study investigators administered the $3 \mathrm{MS}$ examination to the participating patients. The test was a 100-point cognitive scoring system. According to the test interpretation guidelines, normal cognitive function was defined as a $3 \mathrm{MS}$ score between 79 and 100, while a score under 79 was defined as cognitive impairment [37]. We collected the following data for each patient: age, gender, race, highest education level, duration of statin use, associated medical condition(s), blood pressure, serum lipid profile, name and the dose of statin, and 3MS score.

\section{Statistical analysis}

The patient data was entered in the Microsoft Excel (2013, Redmond, WA, USA) spreadsheet. Statistical analysis was done using SPSS (Statistical Package for the Social Sciences, version 15.01, IBM, Armonk, NY, USA). We analyzed the mean $3 \mathrm{MS}$ score based on age, gender and duration and type of statin use between the subgroups, and examined 3MS score with moderate-intensity or high-intensity statin use and other parameters. A descriptive analysis was used to provide information on the sample characteristics. Means (with standard deviations), medians (with IQR) and proportions were presented. Binomial tests were used to compare the sample proportion of cognitive impairment (as indicated by the 3MS score being 79 or under) to the percentage cognitive impairment in the USA (which is 5\%). The sample size of 213 subjects was needed in order to ascertain adequate power for the study, given the inconclusive data results found in the literature and the lack of data concerning the proportion of cognitive impairment within the statin population, and the prevalence of cognitive impairment was based on professional opinion and observation. The patients' education levels and duration of statin use were considered in the statistical analysis through the use of Pearson correlation with the scores of $3 \mathrm{MS}$ scale. Independent $t$-test was used to compare the mean $3 \mathrm{MS}$ score to the gender 
and Pearson correlation was used to examine the relationship between 3MS score with age. We used independent $t$-test to observe the difference between the $3 \mathrm{MS}$ score with moderateintensity or high-intensity statin use, and race, gender and comorbid conditions. In this study, significance was defined as a $\mathrm{P}<0.05$.

\section{Results}

Two hundred thirteen patients participated in the study. Cognitive impairment was observed in $17.8 \%$ of all patients who were either on MIST or on HIST. Subgroup analysis showed that eight patients $(5.7 \%)$ in the MIST group and 30 patients $(41.7 \%)$ in the HIST group had cognitive impairment $(\mathrm{P}<$ $0.001)$.

\section{Baseline characteristics}

About two-thirds of all the patients $(141 / 213 ; 66.2 \%)$ were on MIST while the rest $(72 / 213 ; 33.8 \%)$ were on HIST. The age range of all patients was between 26 and 96 years. The mean age of all the patients was 55.4 years. There were no statistically significant differences in the mean ages between the patients on MIST with normal cognitive function and patients on MIST with cognitive impairment; patients on HIST with normal cognitive function and patients on HIST with cognitive impairment; and patients on MIST with cognitive impairment and patients on HIST with cognitive impairment (Table 1). The majority of the patients with cognitive impairment were in the age group of 50 to 59 years $(48.6 \%)$ (Fig. 1). There was no correlation between $3 \mathrm{MS}$ score and age $(\mathrm{r}=-0.106)$.

Our study had 58.2\% male patients among all the patients who were on statin therapy. There was no statistically significant difference between the mean $3 \mathrm{MS}$ scores of all men and women $(91.2 \pm 9.5$ vs. $91.3 \pm 12.8 ; \mathrm{P}=0.98)$. In the group of patients on the MIST with cognitive impairment, there were more women than men $(62.5 \%$ vs. $37.5 \%$; P $<0.05)$. On the contrary, there were more men than women in the group of patients on the HIST with cognitive impairment (60\% vs. $40 \%$; $\mathrm{P}<0.05$ ) (Table 1).

Overall, the majority of all the patients were Caucasians (36.6\%), while 35.5\% were African-Americans, 14.1\% were Hispanics, and $17.8 \%$ were of other races mainly Asians. There was no statistically significant difference between the mean 3MS scores among all the races (Caucasians 92.9 \pm 9.4 , African-Americans $89.9 \pm 13.5$, Hispanics $88.8 \pm 10.4$, and other races $91.9 \pm 8.9 ; \mathrm{P}=0.207)$. The majority of the patients in the MIST with cognitive impairment group were Caucasians and African-Americans (37.5\% each), followed by Hispanics and other races $(12.5 \%$ each), while the majority of the patients in the HIST with cognitive impairment group were African-Americans (36.7\%), followed by Hispanics (26.7\%), Caucasians (23.3\%) and other races (13.3\%). There was no statistically significant difference in the race distribution between the patients on MIST with cognitive impairment group and the patients on HIST with cognitive impairment group (Table 1).

\section{Level of education and cognitive function}

There was a weakly positive relationship between the level of education and cognitive function score $(r=0.252)$. Although cognitive impairment was observed more in patients who had an education level beyond high school in both MIST group (87.5\%) and HIST group (73.3\%), the difference between the two groups was not significant $(\mathrm{P}=0.650)$ (Table 1).

\section{Duration of statin therapy and cognitive function}

Our study showed that there were significantly greater proportion of patients $(56.7 \%)$ in the HIST with cognitive impairment group who took statins for more than 10 years compared to $12.5 \%$ patients in the MIST with cognitive impairment group $(\mathrm{P}<0.05)$ (Table 1). There was a negative but weak correlation between the duration of statin therapy and cognitive function score $(\mathrm{r}=-0.283)$ (Fig. 2).

\section{Blood pressure and lipid parameters}

We found no significant difference in the mean blood pressures, mean total cholesterol levels, mean high-density cholesterol (HDL) levels, and mean triglyceride levels between the patients on MIST with normal or impaired cognitive function, between the patients on HIST with normal or impaired cognitive function, and between the patients on MIST or HIST with cognitive impairment. The mean low-density cholesterol (LDL) levels were higher in the patients on MIST with cognitive impairment compared to the patients on MIST with normal cognitive function $(\mathrm{P}<0.05)$, but there was no difference in the mean LDL levels between the patients on HIST with normal or impaired cognitive function, and between the patients on MIST or HIST with cognitive impairment (Table 1).

\section{Comorbid medical conditions}

We found that there was a significantly lower frequency of association of hypertension in the patients on MIST with cognitive impairment group compared to the patients on MIST with normal cognitive function group $(\mathrm{P}<0.05)$, while there was no difference in the frequency of association of hypertension in the patients on HIST with cognitive impairment group compared to the patients on HIST with normal cognitive function group (Table 2). There was a significantly higher frequency of association of hypertension in the patients on HIST with cognitive impairment group compared to the patients on MIST with cognitive impairment group $(\mathrm{P}<0.05)$ (Table 2$)$. We also found that the frequency of association of diabetes mellitus was significantly higher in the patients on HIST with cognitive impairment group compared to the patients on HIST with normal cognitive function group $(\mathrm{P}<0.05)$, while there was no difference in the frequency of association of diabetes mellitus in the patients on MIST with cognitive impairment group compared to the patients on MIST with normal cognitive function 


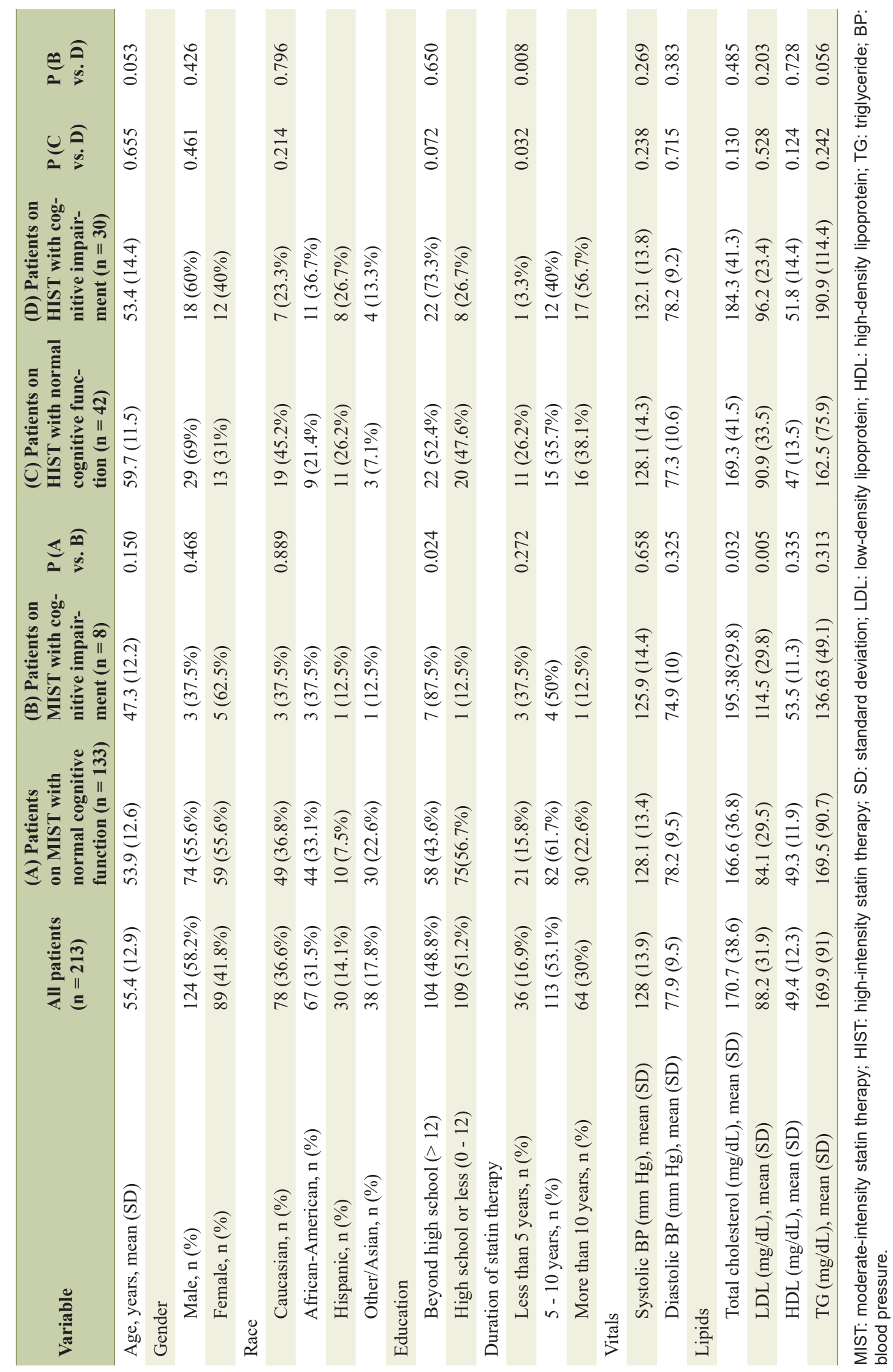




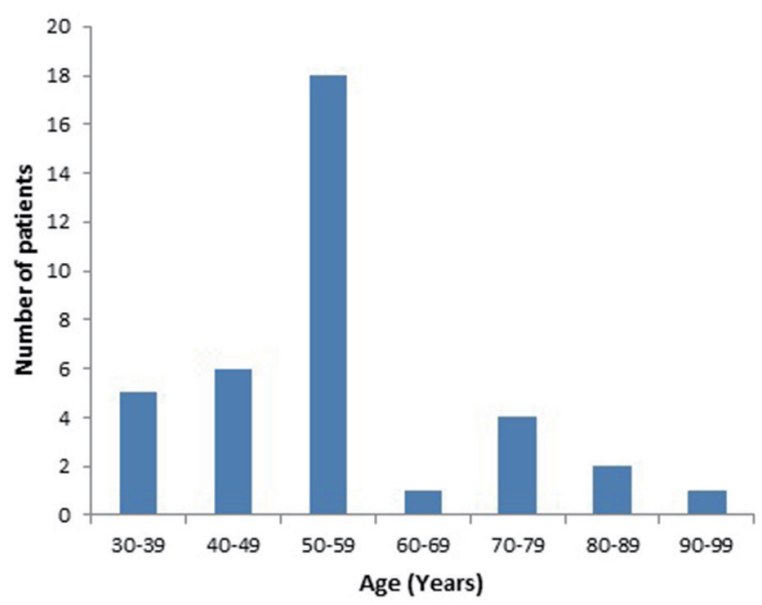

Figure 1. Age distribution of patients with cognitive impairment.

group, as well as between the patients on HIST with cognitive impairment group compared to the patients on MIST with cognitive impairment group (Table 2). There were no differences in the frequencies of association of other comorbid medical conditions across all the groups, such as hypothyroidism, depression, coronary artery disease, cerebrovascular accident, congestive heart failure, chronic obstructive pulmonary disease, chronic kidney disease, anemia, arthritis, malignancy, and obstructive sleep apnea (Table 2). The correlation of 3MS cognitive scores with and without the specific comorbid medical conditions showed no difference between the mean scores of all the groups of patients and in all categories of comorbid medical conditions.

\section{Statin therapy}

Our study showed that the majority of the patients in the HIST group were on atorvastatin $40-80 \mathrm{mg}(71.4 \%$ in normal cognitive function group vs. $66.7 \%$ in cognitive impairment group) compared to the rest of the patients who were on rosuvastatin $20-40 \mathrm{mg}(28.7 \%$ in normal cognitive function group vs. $33.3 \%$ in cognitive impairment group). In the patients on HIST with cognitive impairment group the proportion of patients on atorvastatin $40-80 \mathrm{mg}$ was significantly higher than the proportion of patients on rosuvastatin $20-40 \mathrm{mg}(66.7 \% \mathrm{vs}$. $33.3 \%$; $\mathrm{P}<0.05$ ) (Table 3).

Among the patients who were on MIST with normal cognitive function, simvastatin 20 - $40 \mathrm{mg}$ was the most commonly used statin therapy (29.3\%) followed by atorvastatin $10-20$ $\mathrm{mg}(27.1 \%)$, rosuvastatin $10 \mathrm{mg}(15.8 \%)$, pravastatin $40-80$ $\mathrm{mg}(9 \%)$, lovastatin $40 \mathrm{mg}(6 \%)$, pitavastatin 2 - $4 \mathrm{mg}(5.3 \%)$, fluvastatin XL $80 \mathrm{mg}(4.5 \%)$, and fluvastatin $40 \mathrm{mg}$ twice a day $(3 \%)$. In the group of patients on MIST with cognitive impairment, atorvastatin 10 - $20 \mathrm{mg}$ was the most commonly used statin therapy $(50 \%)$ followed by rosuvastatin $10 \mathrm{mg}$ $(25 \%)$, simvastatin $20-40 \mathrm{mg}(12.5 \%)$ and pravastatin $40-80$ $\mathrm{mg}(12.5 \%)$ (Table 3$)$. There was no significant difference in the proportion of patients on a specific statin type between the patients on MIST with normal cognitive function group and

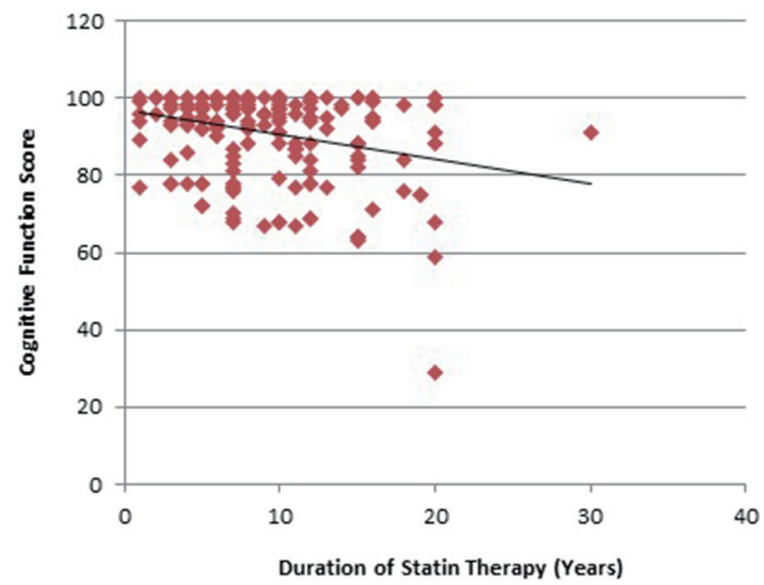

Figure 2. Correlation of duration of statin therapy and cognitive function score.

the patients on MIST with cognitive impairment group, as well as between the patients on HIST with normal cognitive function group and the patients on HIST with cognitive impairment group (Table 3 ).

\section{Discussion}

Our study had three major findings: first, a significantly higher association of cognitive impairment in patients who were on HIST compared to the general population; second, a weakly negative correlation between the duration of statin therapy and cognitive function score; and third, cognitive impairment was most commonly associated with moderate- or high-intensity atorvastatin therapy.

Several studies have reported that the prevalence of cognitive impairment in the US population above the age of 65 years range between $10 \%$ and $37.4 \%$ [38-41]. Although the actual prevalence of cognitive impairment in the US population under the age of 60 years is not known, several studies with small sample size suggest prevalence rates of $0-13.7 \%$ in adults under 65 years of age [42-45]. In our study, the majority of the patients were under 65 years of age with a mean age of 55.4 years, and the prevalence of cognitive impairment was $17.8 \%$, which was significantly higher compared to the general population as reported in the aforementioned studies. Also, in our study we did not find a correlation between the cognitive function score and age.

There are several prospective and retrospective cohort studies that have reported similar associations between statins and cognitive impairment and other psychological disorders, such as an increased risk of suicide [7-9, 21-23]. As early as in 1997, there have been implications of statin therapy with cognitive behavioral changes, such as increased violence [23]. Since then, there has been increasing data from case reports, various retrospective and prospective studies, which ultimately lead to the FDA formally recognizing that statins may cause cognitive impairment [7, 9, 11, 19, 23, 46]. Muldoon et al have done multiple studies that tested attention, reaction 


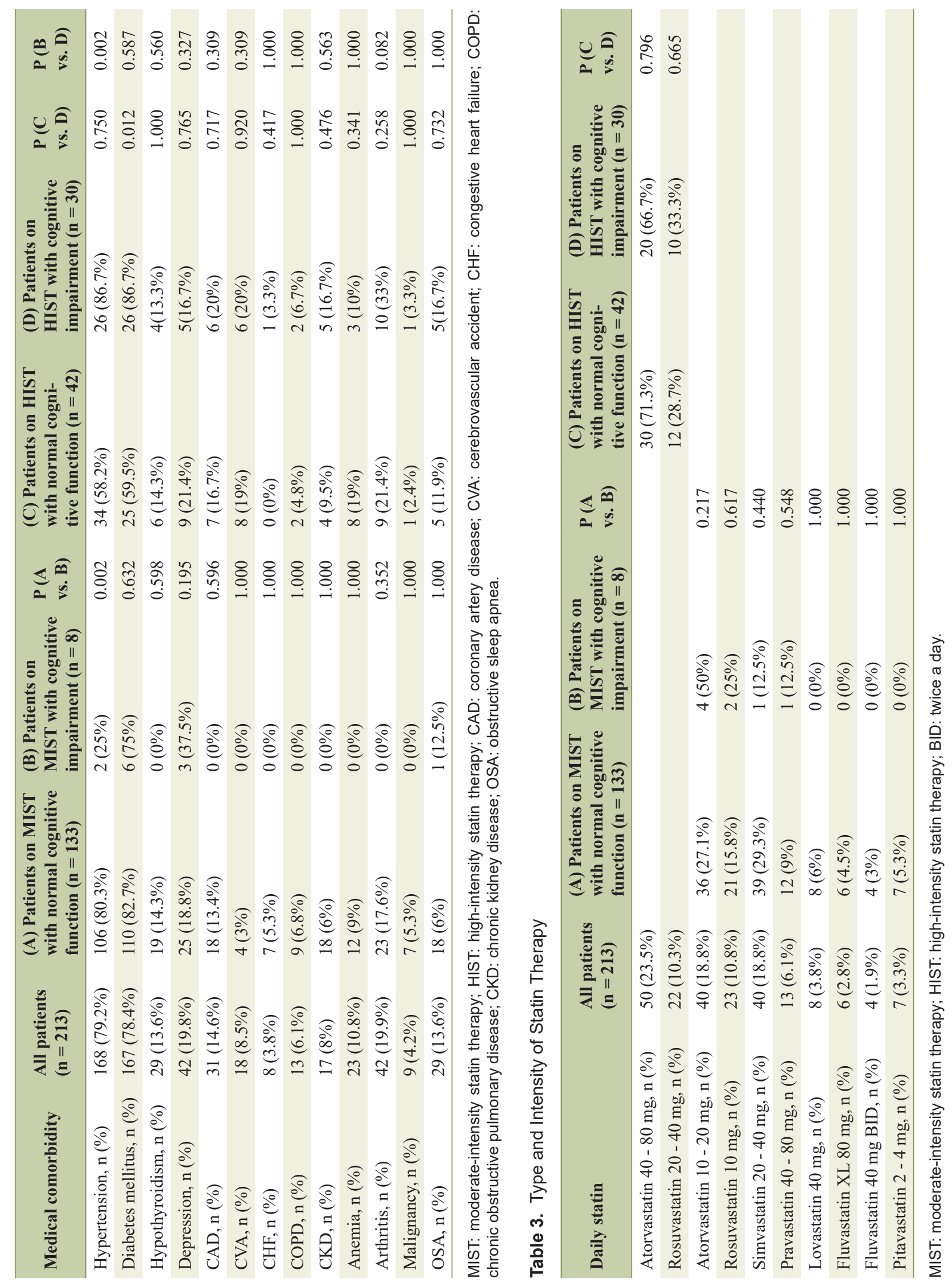


time, and psychomotor speed of those on statins $[7,9]$. Their study showed statistically significant decline in attention, psychomotor speed, and reaction time in those who were on statin therapy compared to placebo. In 2014, the FDA released a report via the Adverse Event Reporting System (AERS) which reported that the patients experienced ill-defined memory loss, confusion, and foggy thinking after statin exposure $[6,19]$. A retrospective study of 3,500 patients found that patients on statin therapy had a significantly higher association of dementia or cognitive impairment compared to the patients who were not on statins $(39.9 \%$ vs. $18.9 \%)$ [46]. A study by Wagstaff et al also observed cognitive impairment with statin therapy but could not determine the causal relationship [11]. Similarly, Glasser et al studied a national cohort of the US population and found $8.6 \%$ prevalence of cognitive impairment in statin users [47]. A significantly negative cognitive effect with statin use was reported in a study that found that the effects were directly related to the potency of the statins, and the statin therapy offered significantly negative impact in the quality of life of the study participants [8].

Additionally, we believe that although the muscle-related adverse effects are more commonly reported by the patients, the influence of statin on cognitive decline remains largely underreported. Golomb et al reported that patients commonly discussed muscle-related adverse effects with their physicians, and the cognitive status changes were rarely assessed or inquired by the physicians [48]. We believe that this phenomenon underestimates the actual prevalence of cognitive impairment associated with the statins. As such the voluntary reporting to the AERS by the physicians varies between $1 \%$ and $10 \%$, which is considered as extremely low [49]. Extrapolation of the underreported data estimates that annually about 3,000 30,000 adverse effects of cognitive impairments due to statins, especially simvastatin and atorvastatin, were not reported to the AERS [21]. Hence, our finding of significantly higher association of cognitive impairment in patients who were on HIST compared to the general population highlights the importance of an inclusive approach in the adverse effect assessment in the form of periodic assessment of cognitive function of patients who are on statin therapy, especially in patients who are on HIST.

There have been several mechanisms that have been proposed as the possible pathogenesis of the association of cognitive impairment secondary to statins. One mechanism proposed by Ainiyet et al suggests a connection between low serum cholesterol and lower activity of central serotonergic release which influences mood, impulsive behavior, and cognition [22]. Experiments in animal models have shown that lowering the synaptosomal membrane cholesterol with statins results in a decline in the number of serotonin receptors, which leads to a reduction in the behavioral and cognitive responses [50]. Another mechanism proposed is that the adverse effects of statins on cognition could be related to the lipophilicity of the drug and its ability to cross the blood brain barrier.

In our study, we found a weakly negative correlation between the duration of statin therapy and cognitive function score, suggesting that longer duration of statin therapy was associated with declining cognitive function. More than half of our patients on HIST with cognitive impairment group were on a statin for more than 10 years. The PROspective Study of Pravastatin in the Elderly at Risk (PROSPER) study found no difference in cognitive impairment between patients on pravastatin or placebo within the 42-month follow-up [17]. In 2013, a meta-analysis found no significant short-term cognitive detriment and potentially beneficial role of statins in prevention of dementia with long-term use [28]. Our finding of negative correlation between the duration of statin therapy and cognitive function score is unique. Further studies are needed to explore the influence or association of both the dose and duration of statin therapy on cognitive function.

We found that the cognitive impairment was most commonly associated with moderate- or high intensity atorvastatin therapy. A retrospective review of 3,500 patients revealed that among patients on statin treatment with cognitive deficit, the majority of the patients were on atorvastatin $(43.9 \%)$ or simvastatin $(35.1 \%)$ [46]. The study also found that cognitive impairment was significantly higher in the patients who were on lipophilic statins, such as atorvastatin and simvastatin, compared to the hydrophilic statins, such as rosuvastatin and pravastatin [46]. The mechanism of such association has been proposed as the lipophilicity of the statin and its ability to cross the blood brain barrier. In the 2014 study that analyzed reports of cognitive dysfunction related to statin use within the FDA AERS found that hydrophilic statins (rosuvastatin and pravastatin) showed essentially no evidence of increased risk of cognitive dysfunction; however, among lipophilic statins (atorvastatin, simvastatin, fluvastatin, lovastatin, and pitavastatin), those with the highest degree of lipophilicity (atorvastatin, simvastatin) were associated with the highest reporting of associated cognitive impairment $[6,19]$. Evans and Golomb found that the potency of statins had a greater negative effect on cognition [8].

There are several explanations to our findings. Hyperlipidemia inhibits intracellular cholesterol synthesis in the neurons and increases intracellular amyloid $(\mathrm{Ab})$ production. This phenomenon facilitates cell lysis and subsequent inhibition of cholesterol synthesis. Atorvastatin and simvastatin, being lipophilic statins, inhibit neuronal cholesterol promoting neuronal degeneration and subsequent cognitive impairment [51]. Lipophilic statins also increase cerebral levels of interleukin-1 beta and tumor necrosis factor-alpha (TNF-alpha), enhancing neuronal inflammatory response, which results in alteration in the function of neurons [52]. Lipophilic statins also demonstrate passive diffusion and non-selective diffusion into hepatocytes and non-hepatocyte tissues [53-58], while the hydrophilic statins, such as rosuvastatin and pravastatin, are highly hepatoselective and enter into the hepatocytes via active transportation, which makes them less likely associated with nonhepatocytic adverse effects $[51,54]$. Additionally, atorvastatin and simvastatin are metabolized through CY3PA4. Atorvastatin has a longer half-life (about $11-30 \mathrm{~h}$ ), while simvastatin has a higher percentage of absorption (about 65-85\%). Other lipophilic statins, such as fluvastatin, go through an extensive first-pass metabolism and protein binding. It appears to be the reason why atorvastatin and simvastatin have higher association with non-hepatocytic adverse effects, such as cognitive impairment, compared to all other lipophilic statins [51, 54]. Our findings of higher association of cognitive impairment 
with lipophilic atorvastatin were aligned with the other studies that have demonstrated higher association and severity of cognitive impairment with lipophilic statins compared with hydrophilic statins [11, 21, 46, 59-62].

Another finding in our study was a weakly positive correlation between the level of education and cognitive function. This result confirms the association that has been proven after several years of research. Studies have shown that education provides cognitive reserves that are neuroprotective even in the setting of dementia, or organic causes of cognitive decline. Wilson et al found that higher educational levels were related to higher baseline level of cognitive function and there was no linear correlation between educational level and rate of agerelated cognitive decline [63]. Zahodne et al supported these findings in their 12-year longitudinal cohort study on the effect of education on cognitive decline. They concluded that higher educational attainment resulted in a robust baseline cognitive performance, but higher educational attainment did not slow age-related cognitive decline even in individuals older than 70 years [64]. These findings help eliminate educational attainment as a confounding factor in our study.

Among the associations of comorbid medical conditions, our study also demonstrated a higher association of hypertension and diabetes mellitus in the patients on HIST with cognitive impairment. There are many studies that have shown an association between hypertension and cognitive impairment likely due to white matter changes secondary to ischemic microvascular alterations [65]. On the contrary, we also found that there was a significantly lower frequency of association of hypertension in the patients on MIST with cognitive impairment compared to the patients on MIST with normal cognitive function, which happens to be a unique finding that needs further exploration. The association of cognitive impairment in patients with diabetes mellitus has been reported as well, mostly with elevated glycosylated hemoglobin values and with longer duration of diabetes, irrespective of the treatment modality, such as oral hypoglycemic agents or insulin therapy [66]. Interestingly we found no difference in the frequency of association of diabetes mellitus in the patients on MIST with cognitive impairment compared to the patients on MIST with normal cognitive function, which also happens to be a unique finding that needs further exploration. Several pathophysiological mechanisms have been attributed to the cognitive impairment due to diabetes mellitus, such as hyperglycemia, vascular disease, hypoglycemia, insulin resistance, amyloidosis, concomitant hypertension, depression, etc $[67,68]$.

There were a few limitations in our study. A lack of baseline cognitive function scores did not allow us to assess the change in the cognitive function scores after the specific duration of statin therapy. The primary care practice environment and lack of funding did not allow us to administer a more detailed neurocognitive testing during routine patient visits hence the 3MS test was administered. Similarly, the influence of risk factors, such as high body mass index, smoking, alcohol misuse and sedentary lifestyle, could not be ascertained due to a lack of availability of discrete data. The major strength of our study was a highly selective inclusion criterion that allowed us to exclude patients with certain comorbidities which could have influenced their cognitive function assessment.

\section{Conclusions}

We found a significantly higher association of cognitive impairment in patients who were on MIST or HIST compared to the general population. We found no correlation between cognitive function score and age, a weakly positive correlation between the level of education and cognitive function score, and a weakly negative correlation between the duration of statin therapy and cognitive function score. The HIST was associated with a higher frequency of cognitive impairment compared to the MIST.

\section{Acknowledgments}

The authors thank Christine Rickette, RN (study coordinator) for her contribution to this study.

\section{Financial Disclosure}

None to declare.

\section{Conflict of Interest}

None to declare.

\section{Informed Consent}

It was obtained from all patients.

\section{Author Contributions}

SR contributed to the study design, data collection, data analysis and manuscript writing. SHK, NA, JW, AB and PS contributed to the parts of the Discussion section. All authors contributed to the data collection and editing of the manuscript for the intellectual content. KH contributed to the data analysis.

\section{Data Availability}

The authors declare that data supporting the findings of this study are available within the article.

\section{References}

1. Mozaffarian D, Benjamin EJ, Go AS, Arnett DK, Blaha MJ, Cushman M, Das SR, et al. Heart disease and stroke statistics-2016 update: a report from the American Heart Association. Circulation. 2016;133(4):e38-360.

2. Ridker PM. The JUPITER trial: results, controversies, and implications for prevention. Circ Cardiovasc Qual 
Outcomes. 2009;2(3):279-285.

3. Gu Q, Paulose-Ram R, Burt VL, Kit BK. Prescription cholesterol-lowering medication use in adults aged 40 and over: United States, 2003-2012. NCHS Data Brief. 2014;(177):1-8.

4. Stone NJ, Robinson JG, Lichtenstein AH, Bairey Merz CN, Blum CB, Eckel RH, Goldberg AC, et al. 2013 ACC/ AHA guideline on the treatment of blood cholesterol to reduce atherosclerotic cardiovascular risk in adults: a report of the American College of Cardiology/American Heart Association Task Force on Practice Guidelines. Circulation. 2014;129(25 Suppl 2):S1-45.

5. Salami JA, Warraich H, Valero-Elizondo J, Spatz ES, Desai NR, Rana JS, Virani SS, et al. National trends in statin use and expenditures in the US adult population from 2002 to 2013: insights from the medical expenditure panel survey. JAMA Cardiol. 2017;2(1):56-65.

6. U.S. Food and Drug Administration. FDA announces safety changes in labeling for some cholesterol-lowering drugs [Press release]. 2012. Retrieved December 20, 2016, from http://www.fda.gov/NewsEvents/Newsroom/ PressAnnouncements/ucm293623.htm.

7. Muldoon MF, Ryan CM, Sereika SM, Flory JD, Manuck SB. Randomized trial of the effects of simvastatin on cognitive functioning in hypercholesterolemic adults. Am J Med. 2004;117(11):823-829.

8. Evans MA, Golomb BA. Statin-associated adverse cognitive effects: survey results from 171 patients. Pharmacotherapy. 2009;29(7):800-811.

9. Muldoon MF, Barger SD, Ryan CM, Flory JD, Lehoczky JP, Matthews KA, Manuck SB. Effects of lovastatin on cognitive function and psychological well-being. Am J Med. 2000;108:538-546.

10. Orsi A, Sherman O, Woldeselassie Z. Simvastatin-associated memory loss. Pharmacotherapy. 2001;21(6):767769.

11. Wagstaff LR, Mitton MW, Arvik BM, Doraiswamy PM. Statin-associated memory loss: analysis of 60 case reports and review of the literature. Pharmacotherapy. 2003;23(7):871-880.

12. Parker BA, Polk DM, Rabdiya V, Meda SA, Anderson K, Hawkins K, Pearlson GD, et al. Changes in memory function and neuronal activation associated with atorvastatin therapy. Pharmacotherapy. 2010;30:236e-240e.

13. Zamrini E, McGwin G, Roseman JM. Association between statin use and Alzheimer's disease. Neuroepidemiology. 2004;23(1-2):94-98.

14. Beydoun MA, Beason-Held LL, Kitner-Triolo $\mathrm{MH}$, Beydoun HA, Ferrucci L, Resnick SM, Zonderman AB. Statins and serum cholesterol's associations with incident dementia and mild cognitive impairment. J Epidemiol Community Health. 2011;65(11):949-957.

15. Bettermann K, Arnold AM, Williamson J, Rapp S, Sink $\mathrm{K}$, Toole JF, Carlson MC, et al. Statins, risk of dementia, and cognitive function: secondary analysis of the ginkgo evaluation of memory study. J Stroke Cerebrovasc Dis. 2012;21(6):436-444.

16. Benito-Leon J, Louis ED, Vega S, Bermejo-Pareja F. Statins and cognitive functioning in the elderly: a popula- tion-based study. J Alzheimers Dis. 2010;21(1):95-102.

17. Trompet $\mathrm{S}$, van Vliet $\mathrm{P}$, de Craen AJ, Jolles J, Buckley BM, Murphy MB, Ford I, et al. Pravastatin and cognitive function in the elderly. Results of the PROSPER study. J Neurol. 2010;257(1):85-90.

18. Feldman HH, Doody RS, Kivipelto M, Sparks DL, Waters DD, Jones RW, Schwam E, et al. Randomized controlled trial of atorvastatin in mild to moderate Alzheimer disease: LEADe. Neurology. 2010;74(12):956-964.

19. U.S. Food and Drug Administration. FDA Drug Safety Communication: important safety label changes to cholesterol-lowering statin drugs. 2016. Available at: https:/www.fda.gov/drugs/drug-safety-and-availability/ fda-drug-safety-communication-important-safety-labelchanges-cholesterol-lowering-statin-drugs. Accessed January 30, 2020.

20. Zhang J, Liu Q. Cholesterol metabolism and homeostasis in the brain. Protein Cell. 2015;6(4):254-264.

21. Sahebzamani FM, Munro CL, Marroquin OC, Diamond DM, Keller E, Kip KE. Examination of the FDA warning for statins and cognitive dysfunction. J Pharmacovigilance. 2014;2(141):3-9.

22. Ainiyet J, Rybakowski J. [Low concentration level of total serum cholesterol as a risk factor for suicidal and aggressive behavior]. Psychiatr Pol. 1996;30(3):499-509.

23. Kaplan JR, Muldoon MF, Manuck SB, Mann JJ. Assessing the observed relationship between low cholesterol and violence-related mortality. Implications for suicide risk. Ann N Y Acad Sci. 1997;836:57-80.

24. Ott BR, Daiello LA, Dahabreh IJ, Springate BA, Bixby $\mathrm{K}$, Murali M, Trikalinos TA. Do statins impair cognition? A systematic review and meta-analysis of randomized controlled trials. J Gen Intern Med. 2015;30(3):348-358.

25. Bitzur R. Remembering Statins: Do Statins Have Adverse Cognitive Effects? Diabetes Care. 2016;39(Suppl 2):S253-259.

26. McGuinness B, O'Hare J, Craig D, Bullock R, Malouf $\mathrm{R}$, Passmore P. Statins for the treatment of dementia. Cochrane Database Syst Rev. 2010;8:CD007514.

27. Richardson K, Schoen M, French B, Umscheid CA, Mitchell MD, Arnold SE, Heidenreich PA, et al. Statins and cognitive function: a systematic review. Ann Intern Med. 2013;159(10):688-697.

28. Swiger KJ, Manalac RJ, Blumenthal RS, Blaha MJ, Martin SS. Statins and cognition: a systematic review and meta-analysis of short- and long-term cognitive effects. Mayo Clin Proc. 2013;88(11):1213-1221.

29. Simpson RJ, Jr., Mendys P. The effects of adherence and persistence on clinical outcomes in patients treated with statins: a systematic review. J Clin Lipidol. 2010;4(6):462-471.

30. Corrao G, Ibrahim B, Nicotra F, Zambon A, Merlino L, Pasini TS, Catapano AL, et al. Long-term use of statins reduces the risk of hospitalization for dementia. Atherosclerosis. 2013;230(2):171-176.

31. Li G, Larson EB, Sonnen JA, Shofer JB, Petrie EC, Schantz A, Peskind ER, et al. Statin therapy is associated with reduced neuropathologic changes of Alzheimer disease. Neurology. 2007;69(9):878-885. 
32. Vance JE. Dysregulation of cholesterol balance in the brain: contribution to neurodegenerative diseases. Dis Model Mech. 2012;5(6):746-755.

33. Jick H, Zornberg GL, Jick SS, Seshadri S, Drachman DA. Statins and the risk of dementia. Lancet. 2000;356(9242):1627-1631.

34. Kurata T, Miyazaki K, Kozuki M, Panin VL, Morimoto N, Ohta Y, Nagai M, et al. Atorvastatin and pitavastatin improve cognitive function and reduce senile plaque and phosphorylated tau in aged APP mice. Brain Res. 2011;1371:161-170.

35. Wolozin B, Kellman W, Ruosseau P, Celesia GG, Siegel G. Decreased prevalence of Alzheimer disease associated with 3-hydroxy-3-methyglutaryl coenzyme A reductase inhibitors. Arch Neurol. 2000;57(10):1439-1443.

36. Samaras K, Makkar SR, Crawford JD, Kochan NA, Slavin MJ, Wen W, Trollor JN, et al. Effects of Statins on Memory, Cognition, and Brain Volume in the Elderly. J Am Coll Cardiol. 2019;74(21):2554-2568.

37. Teng EL, Chui HC. The Modified Mini-Mental State (3MS) examination. J Clin Psychiatry. 1987;48(8):314318.

38. Langa KM, Levine DA. The diagnosis and management of mild cognitive impairment: a clinical review. JAMA. 2014;312(23):2551-2561.

39. Kurz A, Diehl J, Riemenschneider M, Perneczky R, Lautenschlager N. [Mild cognitive disorder. Questions of definition, diagnosis, prognosis and therapy]. Nervenarzt. 2004;75(1):6-15.

40. Plassman BL, Langa KM, Fisher GG, Heeringa SG, Weir DR, Ofstedal MB, Burke JR, et al. Prevalence of cognitive impairment without dementia in the United States. Ann Intern Med. 2008;148(6):427-434.

41. Plassman BL, Langa KM, Fisher GG, Heeringa SG, Weir DR, Ofstedal MB, Burke JR, et al. Prevalence of dementia in the United States: the aging, demographics, and memory study. Neuroepidemiology. 2007;29(1-2):125-132.

42. Joosten-Weyn Banningh L, Vernooij-Dassen M, Rikkert MO, Teunisse JP. Mild cognitive impairment: coping with an uncertain label. Int J Geriatr Psychiatry. 2008;23(2):148-154.

43. Kumar R, Dear KB, Christensen H, Ilschner S, Jorm AF, Meslin C, Rosenman SJ, et al. Prevalence of mild cognitive impairment in 60- to 64-year-old community-dwelling individuals: The Personality and Total Health through Life 60+ Study. Dement Geriatr Cogn Disord. 2005;19(23):67-74.

44. Schroder J, Kratz B, Pantel J, Minnemann E, Lehr U, Sauer H. Prevalence of mild cognitive impairment in an elderly community sample. J Neural Transm Suppl. 1998;54:51-59.

45. Coria F, Gomez de Caso JA, Minguez L, RodriguezArtalejo F, Claveria LE. Prevalence of age-associated memory impairment and dementia in a rural community. J Neurol Neurosurg Psychiatry. 1993;56(9):973-976.

46. Roy S, Weinstock JL, Ishino AS, Benites JF, Pop SR, Perez $\mathrm{CD}$, Gumbs EA, et al. Association of Cognitive Impairment in Patients on 3-Hydroxy-3-Methyl-Glutaryl-CoA Reductase Inhibitors. J Clin Med Res. 2017;9(7):638-
649.

47. Glasser SP, Wadley V, Judd S, Kana B, Prince V, Jenny $\mathrm{N}$, Kissela B, et al. The association of statin use and statin type and cognitive performance: analysis of the reasons for geographic and racial differences in stroke (REGARDS) study. Clin Cardiol. 2010;33(5):280-288.

48. Golomb BA, McGraw JJ, Evans MA, Dimsdale JE. Physician response to patient reports of adverse drug effects: implications for patient-targeted adverse effect surveillance. Drug Saf. 2007;30(8):669-675.

49. Kessler DA. Introducing MEDWatch. A new approach to reporting medication and device adverse effects and product problems. JAMA. 1993;269(21):2765-2768.

50. Engelberg H. Low serum cholesterol and suicide. Lancet. 1992;339(8795):727-729.

51. Sparks DL, Connor DJ, Browne PJ, Lopez JE, Sabbagh MN. HMG-CoA reductase inhibitors (statins) in the treatment of Alzheimer's disease and why it would be ill-advise to use one that crosses the blood-brain barrier. J Nutr Health Aging. 2002;6(5):324-331.

52. Chauhan NB, Siegel GJ, Feinstein DL. Effects of lovastatin and pravastatin on amyloid processing and inflammatory response in TgCRND8 brain. Neurochem Res. 2004;29(10):1897-1911.

53. Kim MC, Ahn Y, Jang SY, Cho KH, Hwang SH, Lee MG, Ko JS, et al. Comparison of clinical outcomes of hydrophilic and lipophilic statins in patients with acute myocardial infarction. Korean J Intern Med. 2011;26(3):294303.

54. Hamelin BA, Turgeon J. Hydrophilicity/lipophilicity: relevance for the pharmacology and clinical effects of HMG-CoA reductase inhibitors. Trends Pharmacol Sci. 1998;19(1):26-37.

55. Schachter M. Chemical, pharmacokinetic and pharmacodynamic properties of statins: an update. Fundam Clin Pharmacol. 2005;19(1):117-125.

56. Pfefferkorn JA, Song Y, Sun KL, Miller SR, Trivedi BK, Choi C, Sorenson RJ, et al. Design and synthesis of hepatoselective, pyrrole-based HMG-CoA reductase inhibitors. Bioorg Med Chem Lett. 2007;17(16):4538-4544.

57. Corsini A, Bellosta S, Baetta R, Fumagalli R, Paoletti R, Bernini F. New insights into the pharmacodynamic and pharmacokinetic properties of statins. Pharmacol Ther. 1999;84(3):413-428.

58. White CM. A review of the pharmacologic and pharmacokinetic aspects of rosuvastatin. J Clin Pharmacol. 2002;42(9):963-970.

59. Golomb BA, Evans MA, Dimsdale JE, White HL. Effects of statins on energy and fatigue with exertion: results from a randomized controlled trial. Arch Intern Med. 2012;172(15):1180-1182.

60. Thelen KM, Rentsch KM, Gutteck U, Heverin M, Olin M, Andersson U, von Eckardstein A, et al. Brain cholesterol synthesis in mice is affected by high dose of simvastatin but not of pravastatin. J Pharmacol Exp Ther. 2006;316(3):1146-1152.

61. Rojas-Fernandez CH, Cameron JC. Is statin-associated cognitive impairment clinically relevant? A narrative review and clinical recommendations. Ann Pharmacother. 
2012;46(4):549-557.

62. Locatelli S, Lutjohann D, Schmidt HH, Otto C, Beisiegel U, von Bergmann K. Reduction of plasma 24S-hydroxycholesterol (cerebrosterol) levels using high-dosage simvastatin in patients with hypercholesterolemia: evidence that simvastatin affects cholesterol metabolism in the human brain. Arch Neurol. 2002;59(2):213-216.

63. Wilson RS, Hebert LE, Scherr PA, Barnes LL, Mendes de Leon CF, Evans DA. Educational attainment and cognitive decline in old age. Neurology. 2009;72(5):460-465.

64. Zahodne LB, Glymour MM, Sparks C, Bontempo D, Dixon RA, MacDonald SW, Manly JJ. Education does not slow cognitive decline with aging: 12-year evidence from the victoria longitudinal study. J Int Neuropsychol Soc. 2011;17(6):1039-1046.

65. Aronow WS. Hypertension and cognitive impairment. Ann Transl Med. 2017;5(12):259.

66. Roy S, Kim N, Desai A, Komaragiri M, Baxi N, Jassil N, Blessinger $\mathrm{M}$, et al. Cognitive Function and Control of Type 2 Diabetes Mellitus in Young Adults. N Am J Med Sci. 2015;7(5):220-226.

67. Kodl CT, Seaquist ER. Cognitive dysfunction and diabetes mellitus. Endocr Rev. 2008;29(4):494-511.

68. Cosway R, Strachan MW, Dougall A, Frier BM, Deary IJ. Cognitive function and information processing in type 2 diabetes. Diabet Med. 2001;18(10):803-810. 